\title{
Les Goncourt historiens, sous la direction d'Éléonore REVERZY et Nicolas BOURGUINAT
}

\section{Maria Emanuela Raffi}

\section{(2) OpenEdition}

\section{Journals}

\section{Edizione digitale}

URL: https://journals.openedition.org/studifrancesi/12004

DOI: 10.4000/studifrancesi. 12004

ISSN: 2421-5856

\section{Editore}

Rosenberg \& Sellier

\section{Edizione cartacea}

Data di pubblicazione: 1 avril 2018

Paginazione: 159-160

ISSN: 0039-2944

\section{Notizia bibliografica digitale}

Maria Emanuela Raffi, «Les Goncourt historiens, sous la direction d'Éléonore reverzy et Nicolas BOURGUINAT», Studi Francesi [Online], 184 (LXII | I) | 2018, online dal 03 juillet 2018, consultato il 17 novembre 2021. URL: http://journals.openedition.org/studifrancesi/12004 ; DOI: https://doi.org/ 10.4000/studifrancesi.12004

Questo documento è stato generato automaticamente il 17 novembre 2021.

\section{(c) (i) (9)}

Studi Francesi è distribuita con Licenza Creative Commons Attribuzione - Non commerciale - Non opere derivate 4.0 Internazionale. 


\title{
Les Goncourt historiens, sous la direction d'Éléonore REVERZY et Nicolas BOURGUINAT
}

\author{
Maria Emanuela Raffi
}

NOTIZIA

Les Goncourt historiens, sous la direction d'Éléonore REVERZY et Nicolas BOURGUINAT, Presses Universitaires de Strasbourg, 2017, 279 pp.

1 Il volume raccoglie le comunicazioni del convegno realizzato a Strasburgo, con lo stesso titolo, nell'aprile del 2015. Nella «Préface», Éléonore Reverzy approfondisce la concezione della storia da parte dei Goncourt, presa fra un'idea narrativista secondo la celebre formula «l'histoire est un roman qui a été» e una scrittura «qui fragmente et sectionne le narratif ou le ramène à des emblèmes», fondando la storia su singoli casi e individui e sul culto dell'inedito e del raro.

2 «Une petite histoire?», prima delle quattro parti del volume, è inaugurata dallo studio di Pierre-Jean DUfIEF (Les Goncourt et l'archive vivante), in cui viene messo in risalto il ruolo, interpretato dagli stessi autori, dell'«historien collectionneur [...] dénicheur d'inédits», che costruisce pazientemente un archivio, ma aspira anche a ridare vita a ciò che è passato e apparentemente morto: «la lettre, archive toujours vive, ranime le récit historique». Erika WICKY prende in esame nel suo articolo, Les femmes au XVIII siècle selon les Goncourt: détails, anecdotes et parfums, la tensione fra l'aneddoto e la visione generale della storia, particolarmente evidente in due opere dei Goncourt sulle donne La Femme au XVIII siècle e Les Maitresses de Louis XV - dato che per i due scrittori le donne fanno parte di una zona particolare della storia, «l'histoire intime qui s'intéresse à la vie privée, à l'espace domestique ainsi qu'au corps et aux manœuvres amoureuses». «Mémorialistes plutôt qu'historiens» i Goncourt del Journal appaiono a José-Luis DIAZ (L'histoire littéraire au jour le jour) sotto due aspetti fondamentali: come testimoni della 
vita letteraria «au jour le jour» frammentata in aneddoti e reportages fissati momento per momento e come «sociologues de la littérature», particolarmente attenti ai fenomeni temporali e generazionali legati all'attività letteraria. «Leur vision d'ensemble du champ littéraire - conclude Diaz - s'exprime 'en situation', selon leur propre inscription dans le champ». Un tema particolare è affrontato dallo studio di Michael ROSENFELD - La figure du "pédéraste» dans le "Journal" des Goncourt (1851-1970), che esamina comparativamente i cambiamenti introdotti in tema di omosessualità dalla versione rivista dal solo Edmond e pubblicata nel 1887, quando il peso della censura sulle opere letterarie era diventato più inquietante. Accanto a modifiche e soppressioni, appare interessante il «déplacement» che sposta l'omosessualità nell'Antichità o la attribuisce a personaggi stranieri.

In «Matériaux et méthodes d'une histoire renouvelée», seconda parte del volume, viene ripreso il tema delle donne del XVIII secolo viste dai Goncourt. Nicolas BOURGUINAT ( $L a$ femme au XVIII' siècle, un terrain d'enquête pionnier?) mostra con un consistente numero di esempi come gli studi sulle donne nella storia abbiano avuto un notevole incremento intorno al 1850, attraendo autori celebri quali Victor Cousin, Sainte-Beuve e poco dopo Michelet nonché l'«historiennes des femmes» Clarisse Bader e, naturalmente, i Goncourt con il loro saggio La Femme au xVIII siècle, "couronnement [...] d'un effort méthodique pour saisir la réalité d'une société par les mœurs et les mentalités». Una simile concezione della storia costringe tuttavia gli autori a un enorme allargamento delle fonti ed è su questo problema che verte la riflessione di Marine LE BAïL in La bibliographie des Goncourt au service du XVIII siècle: entre conservation et recréation. In effetti, ciò che i Goncourt hanno costituito è una vera e propria "collection dix-huitiémiste» che l'A. colloca «au confluent de la collection bibliographique et de l'investigation documentaire», destinata a non essere mai completa, ma strumento straordinario per «revivifier» la storia. La storia del teatro è indubbiamente un aspetto della «collection dix-huitiémiste» dei Goncourt e Guy DUCREY (Les Gestes disparus. Les Goncourt pour une autre histoire du théâtre) mette in risalto come, ancora una volta, siano per i Goncourt singoli elementi come i ritratti delle grandi attrici e la loro gestualità a formarne il tessuto: «Au théâtre, la lente déclamation, la grâce nouvelle d'un pas, sont aussi des gestes créateurs qui réorganisent toute une société». In Les Goncourt face à l'histoire religieuse anticléricale Émilie SERMADIRAS sposta l'attenzione sulla «storia religiosa» tracciata nel Journal, divenuta ormai, nella seconda metà dell'ottocento, storia anticlericale e demistificazione desacralizzante. La religiosità intesa quasi come patologia medica appare nei romanzi dei Goncourt, ma nel Journal lo sforzo è quello di mantenere un difficile equilibrio critico fra «le dogmatisme de l'Église [et] l'athéïsme forcené, fondé sur une incrédulité généralisée».

«Les Goncourt historiens des letteres et des beaux arts» è l'argomento della terza parte, dove appare anzitutto lo studio di Peter VANTINE, La Révolution et l'art chez les Goncourt, in cui l'A. rileva il giudizio estremamente negativo dei Goncourt sulla Rivoluzione, soprattutto in quanto causa principale della «dégradation de l'art», inquinata ovunque dalla "passion idéologique». L'arte infatti può essere rivoluzionaria solo sul piano estetico, obiettivo realizzato in parte, per gli autori del Journal, solo da David e Chénier. Un altro modo di tracciare una storia dell'arte da parte dei Goncourt è per Sirin DADAS quello del romanzo, in particolare Manette Salomon (Une autre histoire de l'art - Manette Salomon), in cui gli autori sovrappongono la fiction (le peintre Cariolis) alla realtà storica: «Leur roman de l'artiste leur permet [...] d'inventer une histoire du milieu 
artistique qui reste proche de la réalité tout en substituant le moment historique de la révolution réaliste de Courbet par la mise en scène d'un contre-scandale fictif: celui de l'orientalisme de Cariolis». Écrire l'orientalisme avec les Goncourt di Christine PELTRE rimane nello stesso ambito di riflessioni, sottolineando l'influenza del pittore Charles de Tournemine, già incontrato nell'articolo precedente come ispiratore del personaggio di Cariolis, e il caleidoscopio di immagini orientali e più tardi la presa di posizione critica, ironica, dei Goncourt nei confronti di quello stesso gusto e l'interesse, da collezionisti, per i soli oggetti d'arte arabo-musulmani. Sébastien ROLDAN sposta l'attenzione sul trattamento del tempo operato dai Goncourt nella loro scrittura - Les Goncourt historiens du temps à l'heure de la modernité littéraire (1856-1861) - e sull'instantané che lo caratterizza. Tre personaggi «déphasés temporellement», vengono studiati come esempi da Roldan: le père Thibault (Une voiture de masques), Charles Demailly (Les Hommes de lettres) e Barnier (Sœur Philomène); per tutti «le temps, malgré les apparences, n'avance pas linéairement [...], il échoppe sur un présent divisible infiniment et redistribuable, redéfinissable, pulvérisable à tout instant».

5 L'ultima parte del volume - «Histoire morale, histoire sociale» - si apre con il contributo di Federica D'ASCENZO, Les Goncourt ou la vision antilibérale de l'histoire, che mette in evidenza le forti critiche dei Goncourt - «rentiers et propriétaires terriens» al dominio del denaro nella società dell'epoca. Attraverso i diversi romanzi (Charles Demailly, Manette Salomon, Germinie Lacerteux, Les Frères Zemganno) i guasti prodotti dal denaro si estendono dalle arti a tutto il tessuto sociale fino al popolo e soprattutto alla borghesia, che si identifica con il pensiero liberale, «coupable des ravages produits par l'hypertrophie de l'argent», ai quali solo si oppone per i Goncourt «l'art désintéressé». Renée Mauperin è l'oggetto di analisi di Véronique CNOCKAERT ("Car la loi n'est pas le sang». Le nom, la terre, la guerre dans "Renée Mauperin"); in questo romanzo, che traccia la genealogia della famiglia creduta estinta dei Villancourt attraverso cinque secoli di guerre, assedi, battaglie, si concretizza una sorta di «revanche sur les faits, sur la réalité du triomphe de la bourgeoisie, pour ces nostalgiques de l'Ancien Régime que sont les Goncourt». Sophie LUCET (Les Goncourt et le drame historique) si interessa alle sfortunate sorti dell'opera teatrale La Patrie en danger, che scatenano le feroci critiche di Edmond nei confronti dei «drames historiques» in voga (di Dumas, Sardou) e la sua strenua difesa della pièce, accuratamente analizzata dall'A., che tuttavia non può smentire «la leçon historique fondamentalement démoralisante qui en émane, en dépit des promesses de son titre». Les jeux de Chérie: impasse et fracture d'une ligne du temps di Sophie PELLETIER pone al centro del discorso critico l'idea di «héritage», che si tratti di una genealogia, come per l'eroina di Chérie, o di idee e affetti, come per quest'ultimo romanzo di Edmond, che trascina con sé una precisa eredità dei Goncourt: «une histoire intime, personnelle, familiale, sociale, politique, littéraire et même romanesque». Il volume si conclude con la riflessione di Philippe GEINOZ (Usage des États-Unis dans les romans d'Edmond de Goncourt) sull'«américanisation» presente negli scritti di Edmond e soprattutto negli ultimi due romanzi - Les Frères Zemganno e Chérie - ed espressa da personaggi come l'acrobata Trompkins e più sommessamente Diana Peterson. Ciò che le accomuna, oltre alla vitalità, è «la brutalité 'américaine' que le narrateur pointe aussi dans le champ esthétique».

Un'«Annexe», con una nota di Daniel PETER su Le dépôt de l'Académie Goncourt aux Archives municipales de Nancy e una «Postface» di Éléonore REVERzY, chiudono il volume. 